\title{
Considerable Size Reduction of Dipole Antenna with a Compact and Broadband Balun
}

\author{
Heeje Han*, Hyungpyo Kim* and Hongjoon Kim ${ }^{\dagger}$
}

\begin{abstract}
A compact and broadband balun is designed using a conventional right-handed transmission line (RHTL) and a left-handed transmission line (LHTL) with lumped elements only. The proposed balun maintains a phase difference of $180^{\circ} \pm 12^{\circ}$ at the output ports for the frequency range of $1.27 \mathrm{GHz}-2.69 \mathrm{GHz}$. Nevertheless, the circuit size is merely $9.5 \mathrm{~mm} \times 12.7$ $\mathrm{mm}$. To verify the performance of the proposed balun, we attached it to a dipole-type antenna and observed that the return loss is greater than $10 \mathrm{~dB}$ for the frequency range between $1.86 \mathrm{GHz}$ and $2.57 \mathrm{GHz}$. The radiation pattern and peak gain are similar to those of the dipole antenna with a conventional balun.
\end{abstract}

Keywords: Compact, Broadband, Balun, Right-handed, Left-handed, Transmission line, RHTL, LHTL, Dipole antenna, Metamaterial

\section{Introduction}

A balun converts an unbalanced signal into a balanced signal and is an essential element in many microwave circuits. To be useful in modern communication circuits and systems, baluns need to be compact and should have broadband characteristics. Instead of using a long transmission line with a narrow bandwidth and a large size, the authors developed a lumped element Wilkinsontype balun to minimize the circuit size [1].

Recently, the microwave applications of left-handed transmission lines (LHTLs) have attracted wide attention [2-8]. Unlike conventional right-handed transmission lines (RHTLs), LHTLs show negative phase propagation, which is a property of metamaterials. Using this interesting property, it is possible to design a broadband Wilkinsontype balun. However, previous metamaterial broadband baluns have a microstrip-based Wilkinson divider with a composite right/left-handed (CRLH) transmission line [4-8]. Therefore, a large area is required to implement these structures.

In this study, we used synthetic RHTLs and LHTLs to implement a balun while minimizing circuit size and maintaining a broad bandwidth. We then attached this compact balun to a dipole antenna and verified the resulting antenna performance. For comparison, we also fabricated a dipole antenna with a conventional microstripbased balun.

$\dagger \quad$ Corresponding Author: Dept. of Electrical Engineering, Kyungpook National Univerity, Korea. (hongjoon@knu.ac.kr)

* Dept. of Electrical Engineering, Kyungpook National University, Korea. (\{hjhan,hpkim\}@knu.ac.kr)

Received: December 16, 2015; Accepted: July 6, 2016

\section{Theory of the Broadband Balun}

\subsection{Synthetic RHTL and LHTL theory}

As shown in Fig. 1, an RHTL unit section has a low pass filter structure consisting of two series inductors and a shunt capacitor. In contrast, an LHTL unit section has a high pass filter structure consisting of two series capacitors and a shunt inductor.

When several identical RHTL sections are cascaded, a periodic cutoff frequency appears (the Bragg cutoff frequency), which can be calculated as follows [3]

$$
f_{\text {Bragg }}^{R}=\frac{1}{\pi \cdot \sqrt{L_{R} C_{R}}}
$$

The characteristic impedance can be approximated with Eq. (2) [3].

$$
Z_{0 R}=\sqrt{\frac{L_{R}}{C_{R}}}
$$

The phase constant of an RHTL is given by Eq. (3) [2].
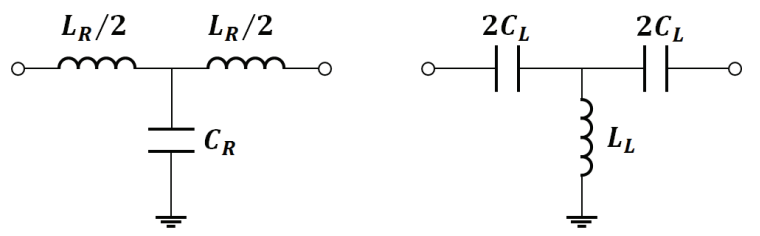

Fig. 1. Unit section of an RHTL (left) and an LHTL (right). Cascading several sections constructs an RHTL and an LHTL 


$$
\beta_{R}=\omega \cdot \sqrt{L_{R} C_{R}}
$$

Similarly, when several identical LHTL sections are cascaded, a Bragg cutoff frequency arises [3].

$$
f_{\text {Bragg }}^{L}=\frac{1}{4 \pi \cdot \sqrt{L_{L} C_{L}}}
$$

The characteristic impedance of an LHTL can be approximated with Eq. (5) [3].

$$
Z_{0 L}=\sqrt{\frac{L_{L}}{C_{L}}}
$$

The phase constant of an LHTL is given by Eq. (6) [2].

$$
\beta_{L}=-\frac{1}{\omega \cdot \sqrt{L_{L} C_{L}}}
$$

Unlike an RHTL, the phase propagation constant of an LHTL is a negative value. This implies that phase propagation is in the direction opposite to that of the Poynting vector $[3,4]$.

\subsection{Broadband and constant phase differentiation}

Our fabricated balun consists of a Wilkinson power divider based on a synthetic RHTL and a broadband $180^{\circ}$ phase differentiation circuit based on both an RHTL and an LHTL, as shown in Fig. 2.

Designing a Wilkinson power divider with a synthetic RHTL requires a $90^{\circ}$-phase-propagation synthetic line to replace the $\lambda / 4$ lines at a given frequency with an impedance of $\sqrt{2} Z_{0}$. The inductor/capacitor ratio of the RHTL can be obtained from Eq. (2). One has to find the value of the lumped elements from Eq. (3) and identify its cut-off frequency using Eq. (1). Repeating this procedure, the number of unit cells and the values of the lumped

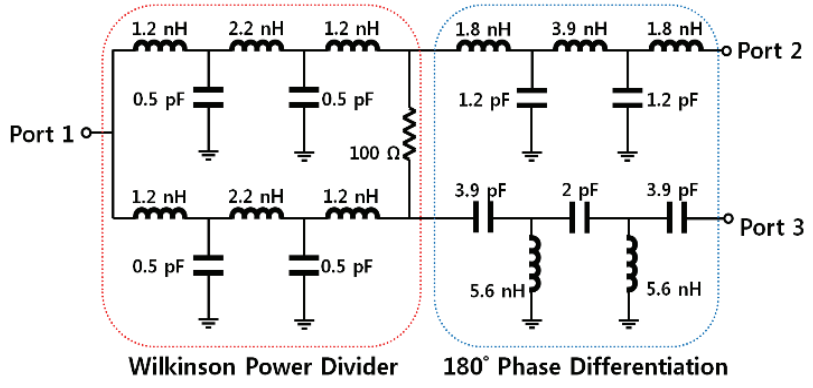

Fig. 2. Schematic diagram of the broadband balun. It consists of a Wilkinson power divider for an equal power split and a phase differentiation section to obtain a constant phase difference between port 2 and port 3

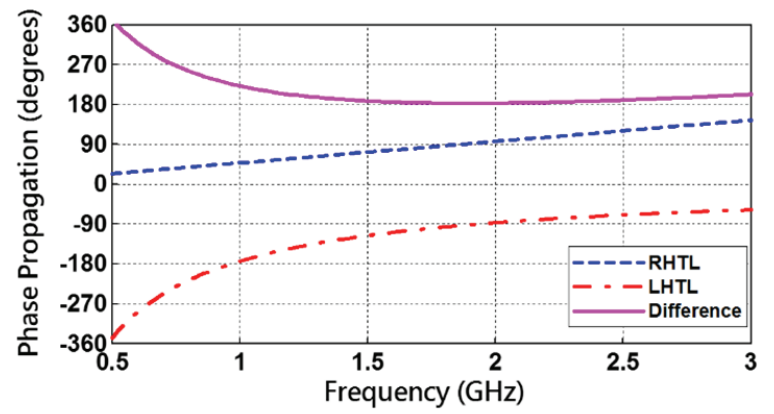

Fig. 3. Theoretical phase propagation of the synthetic RHTL and LHTL, and their difference for the circuit in Fig. 2. The phase difference remains constant around $2 \mathrm{GHz}$

elements can be set. We selected two unit cells of the power divider by considering the phase constant and cutoff frequency. Its characteristic impedance we designed is $67.8 \Omega$.

In the same way, we designed the RHTL of the phase differentiation, and found the values of the LHTL with a $180^{\circ}$ phase difference at $2 \mathrm{GHz}$ using Eqs. (4)-(6). The calculated characteristic impedances of the RHTL and LHTL are $55.9 \Omega$ and $53.3 \Omega$, respectively.

Fig. 3 shows the theoretical phase propagation of each transmission line for a broadband $180^{\circ}$ phase differentiation between the outputs in Fig. 2. As can be seen in Fig. 3, the bandwidth corresponding to the $180^{\circ}$ phase difference between outputs is very broad.

\section{Fabrication and Performance Analysis}

\subsection{Fabrication of antennas with the baluns}

We fabricated two dipole antennas, on FR4 boards with a $1.6 \mathrm{~mm}$ thickness and a relative permittivity of 4.4 : one with the proposed compact broadband balun, and a second one with the conventional balun, as shown in Fig. 4. To fabricate the compact and broadband balun, we used Murata Manufacturing Co.'s chip inductors and Walsin Technology Co.'s chip resistors and capacitors. Their values can be seen in Fig. 2.

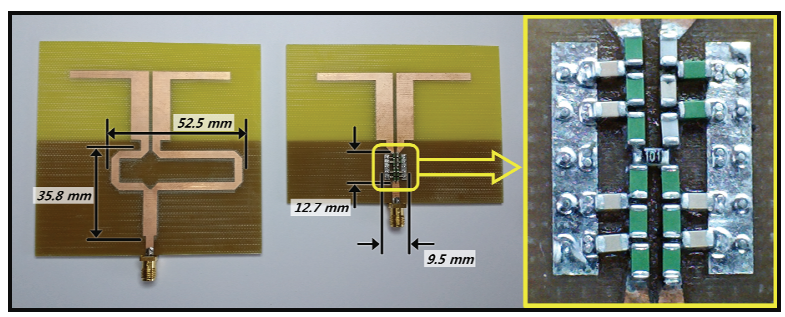

Fig. 4. Two fabricated dipole antennas with the conventional balun (left) and broadband balun (right). The broadband balun with lumped elements is much smaller 


\subsection{Performance of the fabricated baluns}

Before we combined the baluns with the dipole-type antennas, we separately tested the performance of each balun. The target frequency of the baluns is approximately $2 \mathrm{GHz}$. The circuit size of the conventional balun using only a microstrip line is $52.5 \mathrm{~mm} \times 35.8 \mathrm{~mm}$, whereas the proposed broadband balun has a circuit size of only 9.5 $\mathrm{mm} \times 12.7 \mathrm{~mm}$.

Over the frequency range of $1.32 \mathrm{GHz}-2.9 \mathrm{GHz}$ range, the insertion loss $\left(\mathrm{S}_{21}\right.$ and $\left.\mathrm{S}_{31}\right)$ varies from $3.01 \mathrm{~dB}$ to 4.8 $\mathrm{dB}$ in the conventional balun, and from $3.12 \mathrm{~dB}$ to $4.84 \mathrm{~dB}$ in the broadband balun, as shown in Fig. 5. The slight increase in insertion loss of the proposed balun is caused by the low resistances of the lumped elements.

However, because the broadband balun has a Wilkinson power divider, the return losses at all ports $\left(\mathrm{S}_{11}, \mathrm{~S}_{22}\right.$ and $\left.\mathrm{S}_{33}\right)$ and the isolation $\left(\mathrm{S}_{23}\right)$ between output ports are significantly better than those of a conventional balun. The return loss and isolation of the fabricated broadband balun are both greater than $10 \mathrm{~dB}$ in the $1.32 \mathrm{GHz}-2.9$ $\mathrm{GHz}$ frequency range, whereas the isolation of the conventional balun is less than $9.5 \mathrm{~dB}$. In the case of return losses, only S11 is better in the conventional balun.

Fig. 6 shows a performance comparison between the two baluns. For a phase difference of $180^{\circ} \pm 12^{\circ}$ between outputs, the frequency range of the conventional balun is

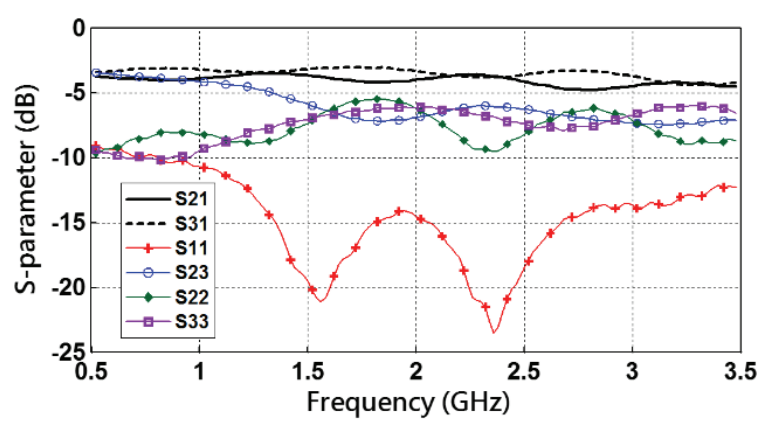

(a)

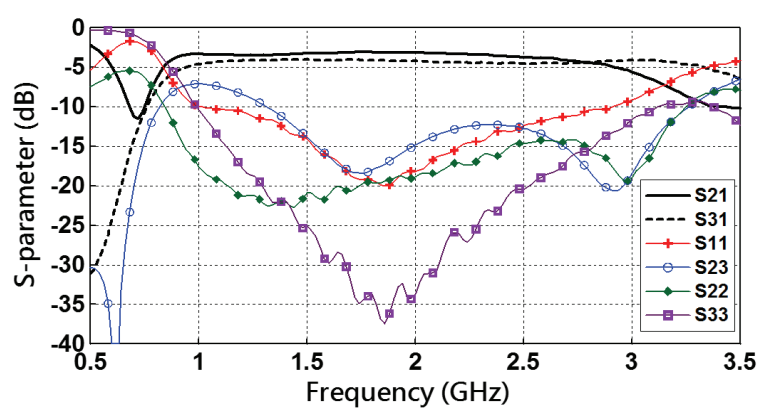

(b)

Fig. 5. Measured magnitude of S-parameters: (a) Conventional balun; (b) Broadband balun. The proposed balun has a good isolation performance because of a Wilkinson divider structure only between $1.82 \mathrm{GHz}$ and $2.1 \mathrm{GHz}$, whereas the broadband balun has a much broader bandwidth, from 1.27 $\mathrm{GHz}$ to $2.69 \mathrm{GHz}$. These measurement results are therefore in close agreement with the theoretical values shown in Fig. 3. Small discrepancies are caused by the parasitics and tolerances of the lumped elements.

Fig. 7 presents the insertion loss and imbalance of both baluns. In the $1.32 \mathrm{GHz}-2.9 \mathrm{GHz}$ frequency range, the insertion loss varies from $0.01 \mathrm{~dB}$ to $1.8 \mathrm{~dB}$ for the conventional balun, and from $0.12 \mathrm{~dB}$ to $1.84 \mathrm{~dB}$ in the case of the broadband balun.

For the same frequency range, the absolute values of the

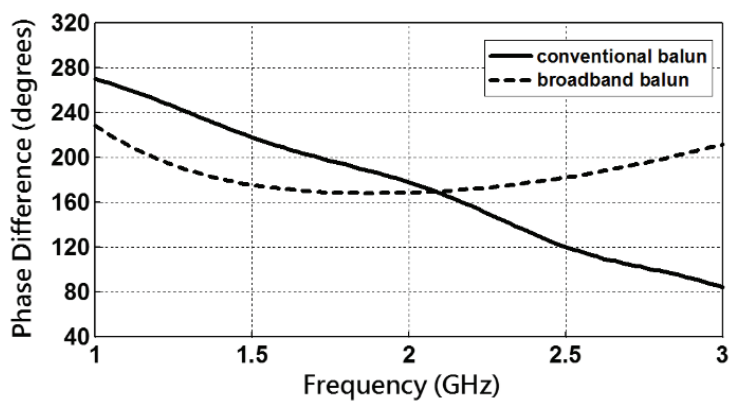

Fig. 6. Measured phase difference between the outputs of the baluns. The proposed balun has a broad constant phase difference, whereas the conventional balun can only maintain a $180^{\circ}$ phase difference within a narrow band.

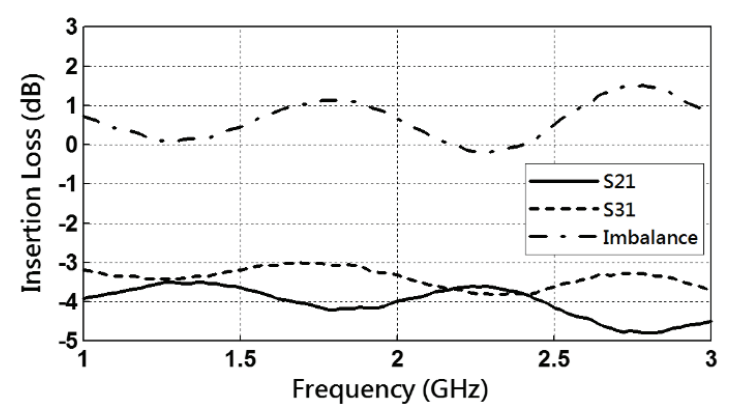

(a)

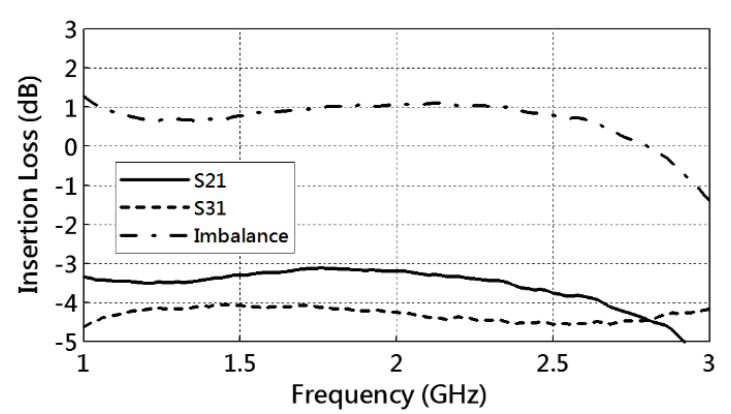

(b)

Fig. 7. Insertion loss and imbalance: (a) Conventional balun; (b) Broadband balun. The broadband balun shows a flat imbalance when compared to that of the conventional type balun 


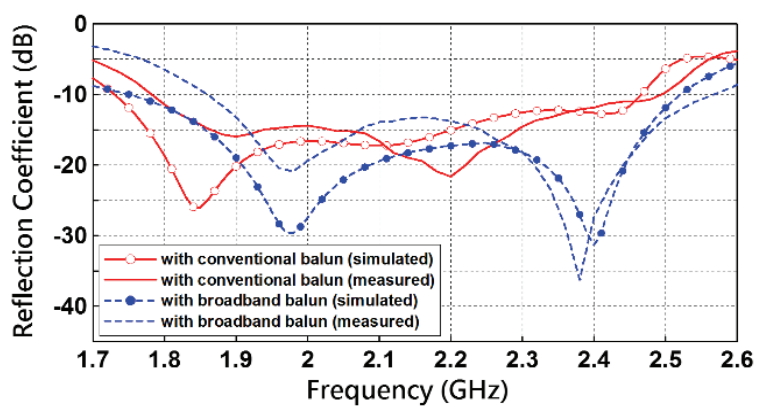

Fig. 8. Magnitude of the antenna reflection coefficients. The simulated and measured results are in good agreement

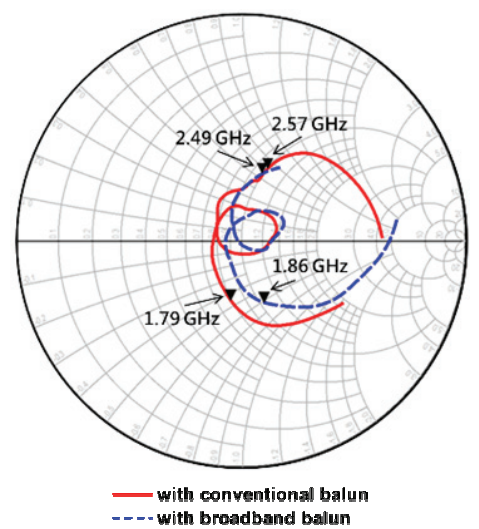

Fig. 9. Measured antenna input impedances. Both impedances trace a similar path on Smith chart

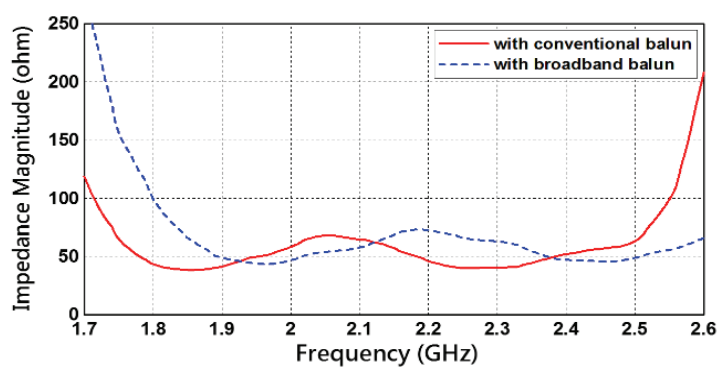

Fig. 10. Measured input impedance magnitude of the antennas. Both antennas show a similar magnitude, although with a slight difference in bandwidth

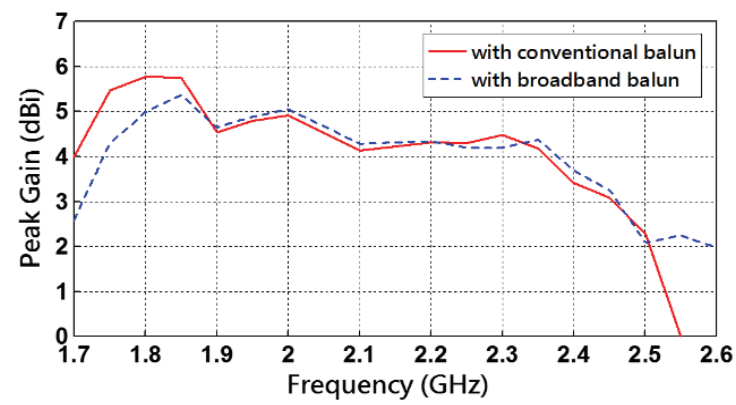

Fig. 11. Measured antenna peak gains. Both peak gains range similarly from $2.09 \mathrm{dBi}$ to $5.05 \mathrm{dBi}$ in the $1.9 \mathrm{GHz}-2.49 \mathrm{GHz}$ band magnitude imbalance between the outputs are less than $1.51 \mathrm{~dB}$ for the conventional balun, and $1.41 \mathrm{~dB}$ for the broadband balun. Therefore, the proposed balun shows the smaller variation of magnitude imbalance between outputs.

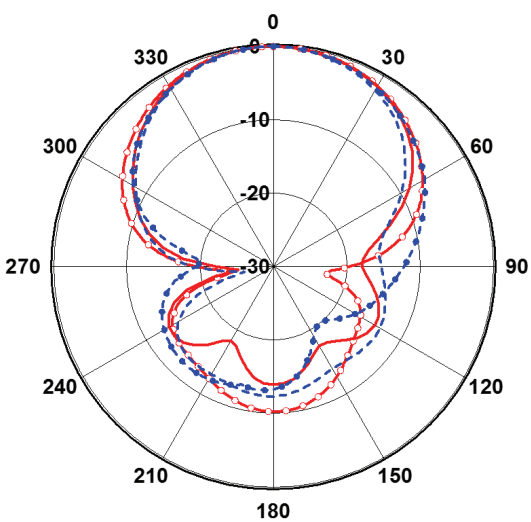

- with conventional balun (simulated) - - with broadband balun (simulated) with conventional balun (measured) ---- with broadband balun (measured)

(a)

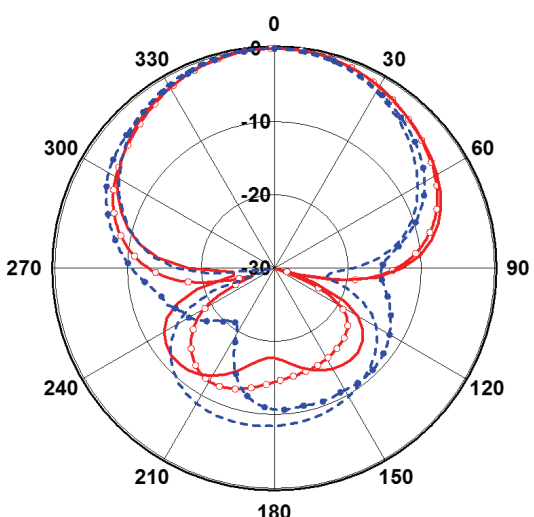

- with conventional balun (simulated) - - with broadband balun (simulated) with conventional balun (measured) ---- with broadband balun (measured)

(b)

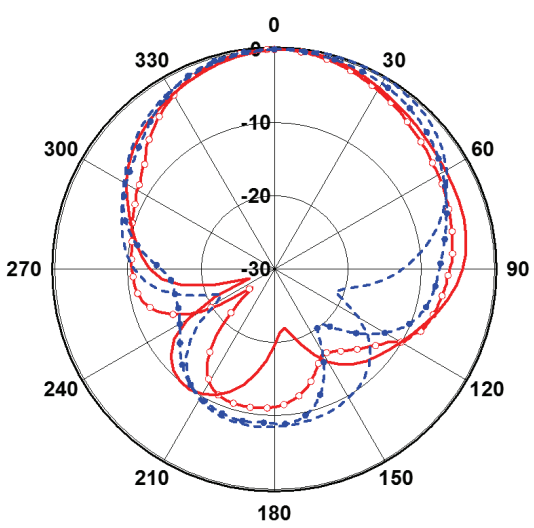

- with conventional balun (simulated) - - with broadband balun (simulated) — with conventional balun (measured) -.--- with broadband balun (measured)

(c)

Fig. 12. Radiation patterns (E-plane) of both antennas at (a) $1.95 \mathrm{GHz}$, (b) $2.2 \mathrm{GHz}$, and (c) $2.45 \mathrm{GHz}$. Both antennas feature similar radiation patterns within the antenna bandwidth 


\subsection{Performance of the dipole antenna with the baluns}

Referring to [9], we designed broadband dipole antennas, combined them with a conventional balun and a broadband balun, and measured their performances. As shown in the measurement results of Fig. 8 , the $10 \mathrm{~dB}$ bandwidth of the antenna with the conventional balun is $0.7 \mathrm{GHz}$ (from 1.79 $\mathrm{GHz}$ to $2.49 \mathrm{GHz}$ ) and that of the broadband balun is 0.71 $\mathrm{GHz}$ (from $1.86 \mathrm{GHz}$ to $2.57 \mathrm{GHz}$ ).

Fig. 9 shows the measured input impedances of both antennas in the Smith chart. Within the bandwidth of each antenna, both impedances trace similar paths in the Smith chart. Their magnitudes are 38.34-68.34 $\Omega$ and 43.26$73.06 \Omega$ for the conventional and broadband antenna baluns, respectively, as shown in Fig. 10.

Fig. 11 and Fig. 12 show the peak gain and radiation patterns (E-plane) of the antennas at several frequencies. Both antennas have similar peak gains in the $1.9 \mathrm{GHz}-2.49$ $\mathrm{GHz}$ frequency range. The radiation patterns of the two antennas are also similar at several frequencies (Fig. 12). However, the size of the proposed balun is significantly smaller than that of the conventional balun. As the proposed balun is composed of only lumped elements, it is possible to integrate this circuit with other front-end modules in wireless communication systems. This means that a front-end module IC and a dipole antenna can be directly connected, without considering the balun size.

\section{Conclusion}

In this study, we evaluated the performance of both a compact, broadband balun fabricated with only lumped elements, and a dipole antenna combined with it. For comparison, a dipole antenna with a conventional balun was also fabricated and tested. By reducing the circuit size of the balun, we could reduce the overall antenna size. Nevertheless, both dipole antennas showed similar performances.

As the suggested design method implies only a simple surface mount, its fabrication is much easier. In addition, the broadband balun can be made with a monolithic microwave integrated circuit (MMIC) process, which implies that the circuit size can be further reduced and that it is possible to fabricate the whole antenna as a planar type antenna. This compact broadband balun can be integrated easily with other IC-type circuits, and be applied not only to dipole antennas but also to many microwave circuits that use broadband balanced signals. Examples of such circuits are balanced amplifiers and balanced mixers. These advantages can make the proposed balun extensively useful, by contributing to a simpler circuit design and considerable size reduction.

\section{Acknowledgements}

This research was supported by the Basic Science Research Program through the National Research Foundation of Korea (NRF) funded by the Ministry of Education (NRF-2015R1D1A1A02061454) and InfoCommunication \& Broadcasting Technology Research Program through the Ministry of Science, ICT and Future Planning (B0101-16-1375).

\section{References}

[1] H. S. Nagi, "Miniature lumped element 180 Wilkinson divider," IEEE MTT-S International Microwave Symposium Digest, vol. 1, pp. 55-58, 2003.

[2] H. Kim, A. B. Kozyrev, A. Karbassi, and D. W. van der Weide, "Linear tunable phase shifter using a lefthanded transmission line," IEEE Microwave and Wireless Components Letters, vol. 15, no. 5, pp. 366368, May. 2005.

[3] H. Kim, S.-J. Ho, M.-K. Choi, A. B. Kozyrev, and D.W. van der Weide, "Combined left-and righthanded tunable transmission lines with tunable passband and $0^{\circ}$ phase shift," IEEE Trans Microwave Theory and Techniques, vol. 54, no. 12, pp. 41784184, Dec. 2006.

[4] M. A. Antoniades, and G. V. Eleftheriades, "A broadband Wilkinson balun using microstrip metamaterial lines," IEEE Antennas and Wireless Propagation Letters, vol. 4, pp. 209-212, 2005.

[5] C.-L. Tseng, and C.-L. Chang, "Wide-band balun using composite right/left-handed transmission line," Electronics Letters, vol. 43, no. 21, pp. 1154-1155, Oct. 2007.

[6] C.-C. Lin, and C.-H. Tseng, "Metamaterial-based Wilkinson balun using asymmetrical T-structure power divider for improving phase response flatness," Microwave Conference Proceedings (APMC), pp. 19-21, Dec. 2012.

[7] U. Park, "A Wilkinson-Type Balun Using a Composite Right/Left-Handed Transmission Line," Journal of Information and Communication convergence engineering, vol. 11, no. 3, pp. 147-152, Sep. 2013.

[8] M. Bemani, S. Nikmehr, and H. R. Takfallah. "Dualband microstrip-to-coplanar stripline Wilkinson balun using composite right- and left-handed transmission lines and its application in feeding dual-band bow-tie antenna," IET Microwaves, Antennas \& Propagation, vol. 8, no. 7, pp. 532-540, May. 2014.

[9] Y. Qian, W. R. Deal, N. Kaneda, and T. Itoh, "Microstrip-fed quasi-Yagi antenna with broadband characteristics," Electronics Letters, vol. 34, no. 23, pp. 2194-2196, Nov. 1998. 


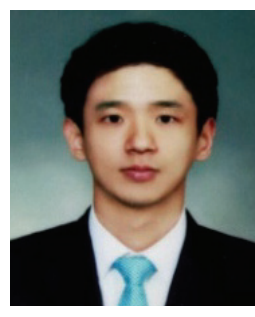

Heeje Han received B.S. degree in Electronic Engineering from Silla University, Korea, in 2013, and M.S. degree in Electrical Engineering from Kyungpook National University, Korea, in 2015. Currently, he is working toward Ph.D. degree in the Department of Electrical Engineering, Kyungpook National University, Korea. His research interests include Microwave circuits and Wireless systems.

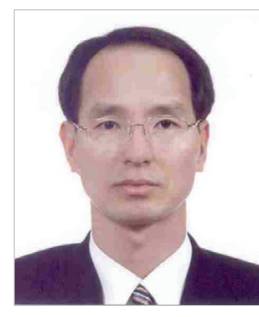

Hyungpyo Kim received B.S., M.S. and Ph.D. degrees in Electrical Engineering from Kyungpook National University, Korea, in 1992, 1994 and 1998, respectively. In 1998, he joined Kyungpook National University, where he is currently a Professor with the Department of Electrical Engineering. His research interests include sensor devices and systems.

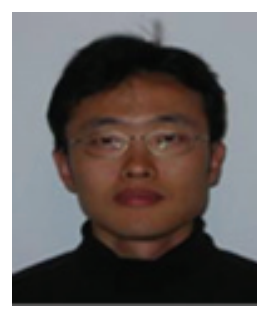

Hongjoon Kim received B.S. degree in Electrical Engineering from Kyungpook National University, Korea, in 1997, and M.S. degree in Electrical Engineering from University of Southern California, USA, in 1999. He received Ph.D. degree in Electrical Engineering from University of Wisconsin-Madison, USA, in 2006. He was an Assistant Professor in the Electrical Engineering Department, City University of New York, USA, from 2006 to 2011. Since 2011, he has worked as an Associate Professor in the Department of Electrical Engineering, Kyungpook National University, Korea. His research interests include RF Communication systems, Electromagnetics, Microwave circuits, Solid state devices and RADAR systems. 